Nonlinear Processes in Geophysics (2005) 12: 129-138

SRef-ID: $1607-7946 / \mathrm{npg} / 2005-12-129$

European Geosciences Union

(c) 2005 Author(s). This work is licensed

under a Creative Commons License.

\title{
Statistical characteristics of irreversible predictability time in regional ocean models
}

\author{
P. C. Chu ${ }^{1}$ and L. M. Ivanov ${ }^{1,2}$ \\ ${ }^{1}$ Naval Ocean Analysis and Prediction Laboratory Department of Oceanography, Naval Postgraduate School Monterey, \\ California, USA \\ ${ }^{2}$ Marine Hydrophysical Institute, Sevastopol, Ukraine \\ Received: 14 September 2004 - Revised: 29 December 2004 - Accepted: 11 January 2005 - Published: 28 January 2005 \\ Part of Special Issue "Nonlinear processes in solar-terrestrial physics and dynamics of Earth-Ocean-System"
}

\begin{abstract}
Probabilistic aspects of regional ocean model predictability is analyzed using the probability density function (PDF) of the irreversible predictability time (IPT) (called $\tau$ PDF) computed from an unconstrained ensemble of stochastic perturbations in initial conditions, winds, and open boundary conditions. Two-attractors (a chaotic attractor and a small-amplitude stable limit cycle) are found in the winddriven circulation. Relationship between attractor's residence time and IPT determines the $\tau$-PDF for the short (up to several weeks) and intermediate (up to two months) predictions. The $\tau$-PDF is usually non-Gaussian but not multimodal for red-noise perturbations in initial conditions and perturbations in the wind and open boundary conditions. Bifurcation of $\tau$-PDF occurs as the tolerance level varies. Generally, extremely successful predictions (corresponding to the $\tau$-PDF's tail toward large IPT domain) are not outliers and share the same statistics as a whole ensemble of predictions.
\end{abstract}

\section{Introduction}

Estimate of predictability skill of regional ocean models is important but difficult. The regional ocean dynamics is sensitive to variability of external forcing such as winds, fresh water discharge, buoyancy flux, and to multi-scale interactions between shelf and abyssal currents (Robinson et al., 1996; Lozano et al., 1996; Jiang and Malanotte-Rizzoli, 1999; Chu et al., 1999a, b, c, d, 2001; Auclair et al., 2003 among others). Bathymetry, coastlines, and physical processes such as wind bursts, fresh water discharge, tides, storm surges, shelf waves, and nonlinear processes affect coastal and abyssal currents and may cause multi-attractor circulation in marginal seas where robust circulation regimes (attractors) are more complicated than simply periodic or even quasi-periodic ones.

Correspondence to: L. M. Ivanov

(lmivanov@nps.edu)
Multiple attractors in atmospheric models are used by several investigators to understand the dynamics of planetary flow regimes (Charney and De Vore, 1979; Molteni, 1996). Multi-modality is found in wind-driven ocean circulation models (Schmeits and Dijkstra, 2000) such as the Kuroshio path variation south of Japan (Masuda et al., 1999). The multi-modality in the probability density function (PDF) of prediction error is caused by the spatial phase organization of the local error growth rate (Miller and Ehret, 2002). Usually, the multi-modality leads to the intermittency of the predictability of atmospheric and ocean models and the formation of non-Gaussian PDF of the prediction error.

Even for prediction error with initial Gaussian PDF, the PDF of predictability time scale is often non-Gaussian with a tail stretching into large predictability time scale values (Chu and Ivanov, 2002, 2005a ${ }^{1}, b^{2}$; Chu et al., 2002a, b; DenholmPrice, 2003). Rare forecasts of enhanced predictability dominate these tails. Obviously, if the occurrences of these predictions increase, the ensemble prediction will be improved.

In this study, a wind-driven circulation model based on the barotropic version of Princeton Ocean Model (POM) (Blumberg and Mellor, 1987) is implemented for a semienclosed rectangular basin with flat bottom to reproduce the multi-attractor regime and to study the variability of model predictability-skill with uncertain initial conditions, external forcing (wind) and open boundary conditions. A recently proposed irreversible predictability time (IPT) $\tau$ (Ivanov et al., 1994; Chu et al., 2002a, b) is taken as a quantitative measure of model predictability skill.

There are three major tasks in this study: (1) to find mechanisms for the formation of non-Gaussian PDF of IPT (called $\tau$-PDF) with a tail stretching into large IPT domain, (2) to detect the effect of stochastic perturbations in external forcing

\footnotetext{
${ }^{1}$ Chu, P. C. and Ivanov, L. M.: Some considerations on stochastic stability of regional oceanic models with perturbed initial conditions, Tellus, revised, 2005a.

${ }^{2}$ Chu, P. C. and Ivanov, L. M.: Some considerations on stochastic stability of regional ocean models with perturbed external forcing, Tellus, in revision, $2005 \mathrm{~b}$.
} 


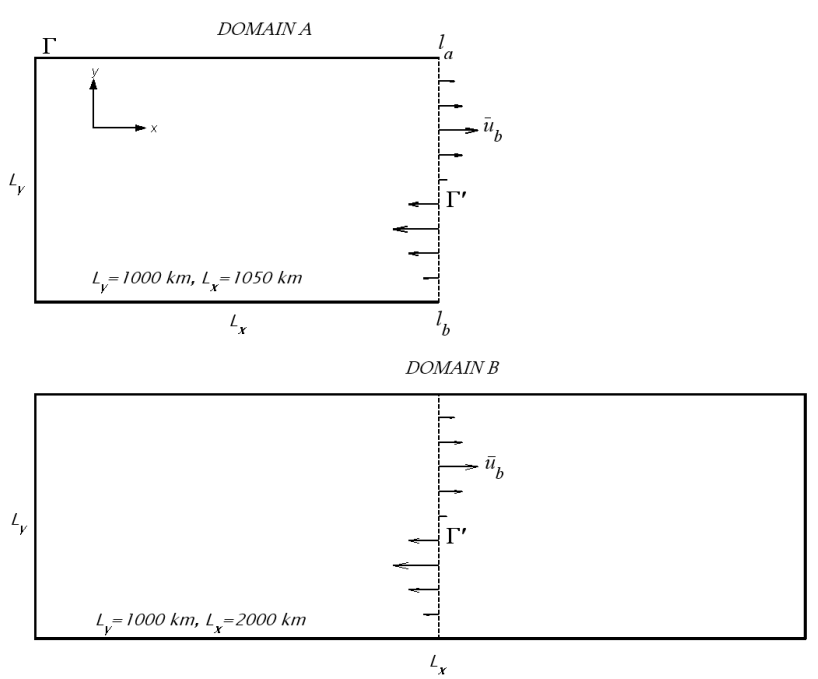

Fig. 1. Two areas for the POM integration: domain-A with three rigid boundaries and the open boundary $\Gamma^{\prime}$ (upper panel), and domain-B with four rigid boundaries (lower panel).

on the model predictability, and (3) to identify the control parameter (or control parameters) for the variability or bifurcation of the model predictability skill such as the change of symmetric $\tau$-PDF into asymmetric $\tau$-PDF with a long tail toward large IPT domain. Fulfilling of these tasks may improve our understanding of the following problems. Do extremely successful predictions (long IPT) share the same statistical properties as predictions of short and intermediate durations? How do the stochastic perturbations in external forcing switch the attractors (noise-induced escapes)? How do they affect a multi-attractor system? What is the most important parameter to determine the $\tau$-PDF characteristics?

The paper is organized as follows. Section 2 describes the reference solution presenting two circulation attractors: a small-amplitude stable limit cycle and a chaotic attractor. Section 3 discusses the method for generating unconstrained ensembles of perturbations added to the initial conditions, wind forcing, and open boundary conditions. Sections 4 and 5 discuss the first and second kinds of predictability. Section 6 presents the conclusions.

\section{The reference solution}

\subsection{Numerical modeling}

\subsubsection{Two domains}

Consider a semi-closed basin with the flat bottom centered at $35^{\circ} \mathrm{N}$ and bounded by three rigid boundaries (DomainA in upper panel of Fig. 1). This basin expands $1000 \mathrm{~km}$ $(1050 \mathrm{~km})$ in the north-south (east-west) direction. The northern, southern, and western boundaries are rigid, and the eastern boundary is open. The Cartesian coordinate system is chosen with the origin at the southwest corner. The $\mathrm{x}$ - and $\mathrm{y}$ - axes point towards the east and north, respectively. The eastern boundary of Domain-A is connected to a domain with four rigid boundaries forming a closed rectangular domain (lower panel of Fig. 1), called Domain-B (Chu et al., 1997).

The circulation reproduced in Domain-B was used to specify open boundary and initial conditions for control run in Domain-A.

\subsubsection{Integration over the closed domain (Domain-B)}

The POM model was integrated for Domain-B with four rigid boundaries from rest and zero surface elevation $(\hat{\xi})$,

$(\hat{u}, \hat{v}, \hat{\xi})=0$,

and forced by the zonal surface pseudo wind stress varying with latitude

$W_{S}=-w_{0} \cos \left(\frac{\pi y}{L_{y}}\right)$,

where $w_{0}=10^{-3} \mathrm{~m}^{2} \mathrm{~s}^{-2}$. The time step is chosen as $2 \mathrm{~min}$. The horizontal resolution is $50 \mathrm{~km}$. The horizontal mixing and bottom stress are parameterized by the bi-harmonic operator and the quadratic drag relation, respectively.

\subsubsection{Integration over the open domain (Domain-A)}

We integrate the POM over Domain-B with four rigid boundaries (non-slip boundary conditions) from the initial conditions (1) and surface forcing (2) and take the solution along the middle of Domain-B $(x=1050 \mathrm{~km})$

$(\bar{u}, \bar{\xi})_{b}=(\hat{u}, \hat{\xi})_{b}$,

as the open boundary condition for the Domain-A integration. The velocity at $x=1050 \mathrm{~km}$ for the Domain-B run is nearly zonal. The solution for the Domain-B integration at day-10 is taken as the initial and open boundary conditions for the control run in Domain-A,

$(\bar{u}, \bar{v}, \bar{\xi})_{t=t_{0}}=\left.(\hat{u}, \hat{v}, \hat{\xi})\right|_{t=10 \text { day }}$.

The model for Domain-A is integrated with the same dissipativity, the wind forcing (2), open boundary condition (3), and from the initial condition (4) for another 750 days. The initial condition is an unclosed single gyre with velocities under $0.35 \mathrm{~m} \mathrm{~s}^{-1}$ and sea surface elevation around 0.05 to $0.1 \mathrm{~m}$ (Fig. 2a). Figure $2 \mathrm{~b}$ shows the normal velocity along the open boundary. The circulation reproduced in Domain-A presents the reference solution.

\subsection{Two-attractor circulation}

Two different attractors in the numerical solutions with the same external forcing and dissipation are detected by the temporal variability of total kinetic energy, Lyapunov dimensionality, and local Lyapunov exponents (Anishenko, 1997).

An attractor can be detected either in the state space (Berloff and McWilliams, 1999) or in the phase space. As 

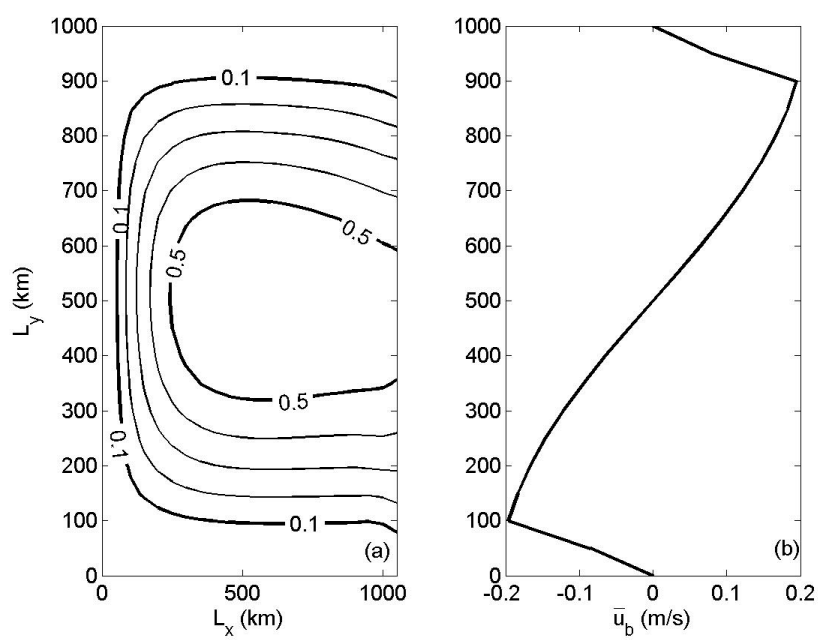

Fig. 2. Initial condition (a) and open boundary condition $\bar{u}_{b}$ (b) for the control run.

indicated in Appendix A, the compressible flow $(u, v)$ with small sea surface elevation can be represented approximately by the geostrophic stream function $\Psi$ (see Eq. A2),

$\Psi(x, y, t)=\Psi_{H}(x, y)+\sum_{k=1}^{K} Z_{k}(t) \Psi_{k}(x, y)$,

where $\Psi_{H}$ is the harmonic function explained in Appendix A.

The orthonormal functions $\left\{\Psi_{k}\right\}$ (modes) generate a Kdimension phase space. The series (5) is truncated at $K=100$. An attractor is characterized by its basin volume and stability.

\subsubsection{Basin volume}

If the model dynamics is high-dimensional and dissipative, it is expected that generally the attractor basin is not a smoothly connected object and has fractal or riddled structure (Guchenheimer and Holmes, 1983). Accordingly to Kaneko (1998), the basin volume of an attractor is estimated as the portion of the randomly selected initial points that are attracted to it. After model integration over 100 days with 10000 initial conditions, the attractor is identified, on which the model trajectory falls. Perturbed model trajectories are approximately distributed between two attractors in the proportion as $1 / 9$.

A manifold of initial conditions $\tilde{Z}_{k}\left(t_{0}\right)$ corresponding to one of the attractors with the basin volume occupied approximately $10 \%$ of phase space (the attractor is identified below as a small amplitude limit cycle) can be specified through smoothing (filtrating) initial conditions accordingly to

$\tilde{Z}_{k}\left(t_{0}\right)=\frac{\lambda_{k}^{2}}{\lambda_{k}^{2}+\chi_{k}^{2}} Z_{k}\left(t_{0}\right)$,

where the function $\chi_{k}$ is defined by

$\chi_{k}=\chi_{0} \max \left|Z_{k}\left(t_{0}\right)\right| / Z_{k}\left(t_{0}\right)$,
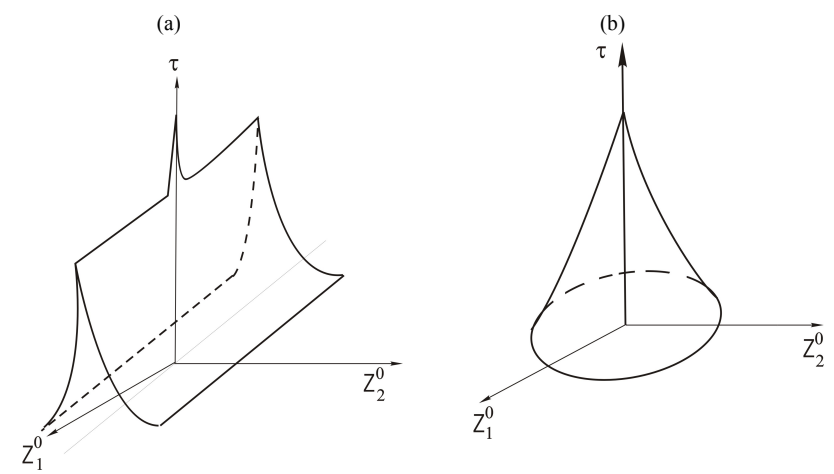

Fig. 3. Typical IPT landscape projected onto a plane $\left\{Z_{1}^{0}, Z_{2}^{0}\right\}$ in phase space: (a) chaotic attractor and (b) limit cycle.

and $\chi_{0}$ is the filter parameter; $\lambda_{k}$ is the eigenvalue corresponding to the basis function $\Psi_{k}(x, y)$. Chu et al. (2003) demonstrate that the filter (6) removes high-order modes and low-order small-amplitude modes.

\subsubsection{Measure of attractor stability}

To estimate attractor stability in forecast metrics

$J(t)=\left\|\Psi^{\prime}\right\|^{2} /\|\bar{\Psi}\|^{2}$,

$\Psi^{\prime}(\mathbf{x}, t)=\Psi(\mathbf{x}, t)-\bar{\Psi}(\mathbf{x}, t), \quad \Psi\left(\mathbf{x}, t_{0}\right)=\Psi_{0}$,

where $(\mathbf{x}, t)$ denotes the spatial and temporal coordinates; $\bar{\Psi}(\mathbf{x}, t)$ and $\Psi(\mathbf{x}, t)$ are the reference solution and andividual prediction, respectively; || || is the Euclidian norm, we use the irreversible predictability time determined as

$\tau=\min \left(t \mid J(t)>\varepsilon_{0}^{2}\right)$,

where $\varepsilon_{0}$ is non-dimensional tolerance level (accepted prediction accuracy).

IPT differs from the e-folding time $\left(\tau_{e}\right)$ or doubling time if prediction error is oscillatory or stochastic. For example, the mean IPT over an ensemble of initial conditions is defined by

$$
\langle\tau\rangle=\left\langle\min \left(t \mid J(t)>\varepsilon_{0}^{2}\right)\right\rangle .
$$

However, the e-folding time is determined by

$$
\left\langle\tau_{e}\right\rangle=\min \left(t \mid\langle J(t)\rangle>\varepsilon_{0}^{2}\right) .
$$

with the corresponding $\varepsilon_{0}^{2}$.

Clearly, unique correlations exist among the landscape of IPT (dependence of IPT on magnitude and orientation of the initial error), the local Lyapunov exponents and the attractor types. Chu and Ivanov $(2005 a, b)^{1,2}$ pointed out that the signs of the local Lyapunov exponents can be determined from the analysis of IPT landscape. 

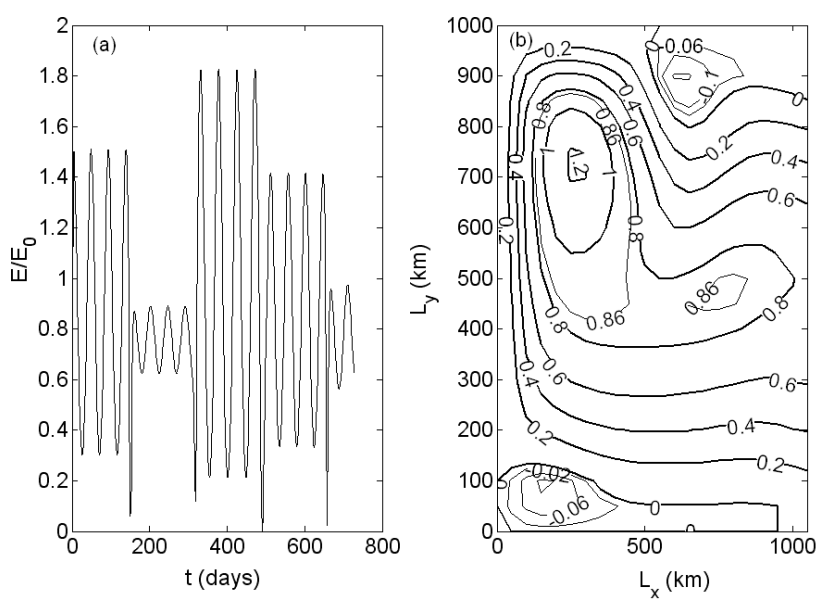

Fig. 4. The chaotic attractor: (a) mean kinetic energy normalized by the value of maximum mean kinetic energy of limit cycle $E_{0}$; (b) snapshot of stream function corresponding to a maximum energy state of attractor.

For a chaotic attractor with at least one positive exponent and one negative exponent, the IPT landscape has a crestlike structure (Fig. 3a). Here, the leading Lyapunov vector is in $\left\{Z_{1}^{0}=Z_{1}\left(t_{0}\right), Z_{2}^{0}=Z_{2}\left(t_{0}\right)\right\}$ plane. If the initial error is oriented perpendicular to the crest, the prediction error grows exponentially. If the initial error is oriented along the crest, it decays or stagnates. A hill-like landscape corresponds to a stable limit cycle (Fig. 3b).

\subsection{Attractor patterns}

\subsubsection{Chaotic attractor}

After integrating the model from initial condition (4) (Fig. 2a), the attractor is characterized by a-periodic behavior of kinetic energy (Fig. 4a). The streamfunction with a maximum energy value (Fig. 4b) shows a strong cyclonic basin-scale gyre with size of about $350 \mathrm{~km}$ (left upper corner) and speed of about $0.9-1.0 \mathrm{~m} \mathrm{~s}^{-1}$. Several smaller size (secondary) eddies occur near the rigid boundary and in the center of the basin. The highest surface elevation reaches $1 \mathrm{~m}$.

The nonlinear regime is identified in the basin by two nondimensional Rossby numbers (Pedlosky, 1987),

$$
R o_{1}=\max \left(\frac{U}{f L}\right) \sim 0.1, \quad R o_{2}=\max \left(\frac{1}{f T}\right) \sim 0.12
$$

where $f \sim 10^{-4} \mathrm{~s}^{-1}$ is the Coriolis parameter; $U \sim 1 \mathrm{~m} \mathrm{~s}^{-1}$ is the characteristic velocity in the basin; $L \sim 100 \mathrm{~km}$ and $T \sim 1$ day are the characteristic spatial and temporal scales of the flow, respectively.

Temporal variation of the kinetic energy averaged over the whole basin (Fig. 4a) shows a-periodic oscillations and transitions between meta-stable states. These oscillations are caused by interactions between the basin-scale cyclonic gyre and the secondary eddies which are generated by barotropic
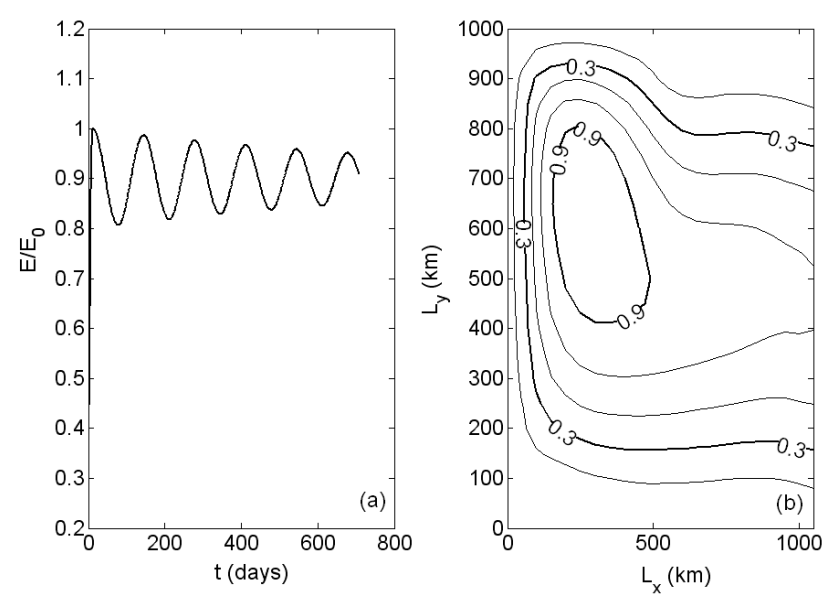

Fig. 5. The small-amplitude limit cycle: (a) mean kinetic energy normalized by its maximum value $E_{0}$, (b) snapshot of stream function averaged over time.

instability of the gyre when the circulation velocity exceeds $0.7-0.8 \mathrm{~m} \mathrm{~s}^{-1}$. The instability produces the secondary eddies disposing along the rigid boundary and in the center of the basin and periodically dissipating due to viscosity damping. Note that for zero horizontal viscosity the chaotic attractor does not develop. Instead an unstable dynamical regime occurs. This case will be discussed in Chu and Ivanov (2005a, b) ${ }^{1,2}$.

The analysis of IPT landscape demonstrates the existence of at least one positive and one negative Lyapunov exponent. It identifies the observed dynamical regime as a chaotic attractor.

\subsubsection{Stable limit cycle}

If the nonlinear filtration procedure (6) is applied to the initial condition (4), an oscillated stable cyclonic gyre quickly develops for the semi-enclosed basin. Figure 5a shows the temporal variation of the kinetic energy averaged over the whole basin. The oscillation period is about 150 days. The nondimensional amplitude of the oscillation is around 0.1 . Figure $5 \mathrm{~b}$ shows temporally averaged stream function.

Such a circulation pattern is represented as a stable limit cycle (small amplitude) in the phase space. This limit cycle is identified using the method proposed by Meacham and Berloff (1997).

\section{Unconstrained ensemble of perturbations}

Since the barotropic model is computationally efficient, the stochastic stability of the reference solution to unconstrained perturbations of the initial condition, open boundary condition, and wind forcing is studied using the Monte-Carlo method with 1000 ensemble samples.

Atmospheric ensemble modeling shows that for small samples the initial error can not cover the phase space 
homogeneously. It leads to an unrealistically-weak growth of prediction error variance. Therefore, for short period predictions it requires the best possible knowledge of the initial state in the sub-region of the phase space where the prediction errors are most likely to grow. In different forecast centers, such as the European Centre for Medium-Range Weather Forecasts and the National Centers for Environmental Prediction, the directions corresponding to the maximum error growth in phase space are identified by the singular vectors (Buizza et al., 1999) and the breed vectors (Toth and Kalnay, 1997), respectively.

This study utilizes a simple Monte-Carlo technique for the three reasons. First, little difference in the statistics is found among ensembles of 1000, 5000, 10000 and 20000 samples. The numerical experiments with 1000 ensemble sample are reliable. Second, a Latin hypercube sampling procedure (LHST, 2001) is used to increase the degree of uniform coverage of the phase space by initial errors. Third, a non-parametrical technique using the Epanechinikov kernel is used to reconstruct PDF from histogram. Details of such a reconstruction can be found in Good (1996).

\subsection{Perturbations to initial condition}

Sensitivity of the reference solution to Gaussian-type perturbations is commonly studied in the atmospheric and oceanic applications (Robinson et al., 1996). In this study, 2-D stochastic perturbations with the correlation function

$G\left(\mathbf{r}-\mathbf{r}^{\prime}\right)=I^{2} \exp \left[-\frac{\left(\mathbf{r}-\mathbf{r}^{\prime}\right)^{2}}{R^{2}}\right]$

are used. Here, $\mathbf{r}=x \mathbf{i}+y \mathbf{j}$, is the displacement vector; $(\mathbf{i}, \mathbf{j})$ are unit vectors along $\mathrm{x}$ and $\mathrm{y}$ axes; $(R, I)$ are the correlation radius and intensity of the noise. The technical details of generating these noises can be found in Sabel'feld (1991).

\subsection{Perturbations to wind forcing}

Surface winds are perturbed by

$\mathbf{u}^{\prime} \equiv\left[u^{\prime}(x, y, t), v^{\prime}(x, y, t)\right]=\left[\mu_{1}(t), \mu_{2}(t)\right] g(x, y)$,

where $\mu_{1}$ and $\mu_{2}$ are independent white noise processes with zero mean and variance of $\sigma^{2}$. In numerical integration, the noises $\left(\mu_{1}, \mu_{2}\right)$ are introduced every hour. The spatial structure function of errors, $g$, is parameterized by,

$g(x, y)=\alpha\left[\pi \lambda_{x} \lambda_{y} \operatorname{erf}\left(\frac{L_{x}}{2 \lambda_{x}}\right) \operatorname{erf}\left(\frac{L_{y}}{2 \lambda_{y}}\right)\right]^{1 / 2}$

$\exp \left(\frac{\left(x-x_{0}\right)^{2}}{\lambda_{x}^{2}}+\frac{\left(y-y_{0}\right)^{2}}{\lambda_{y}^{2}}\right)$

which describes the impact of the localized atmospheric eddy activity near $\left(x_{0}, y_{0}\right)$ on the surface wind perturbations (Sura et al., 2001). Here, erf is the error function; $\alpha$ is a scaling parameter; $\left(\lambda_{x}, \lambda_{y}\right)$ are the de-correlation scales. Noise in surface winds with $\sigma^{2}=28 \mathrm{~m}^{2} \mathrm{~s}^{-2}$ corresponds to observed typical atmosphere conditions in the North Atlantic region (Wright, 1988).

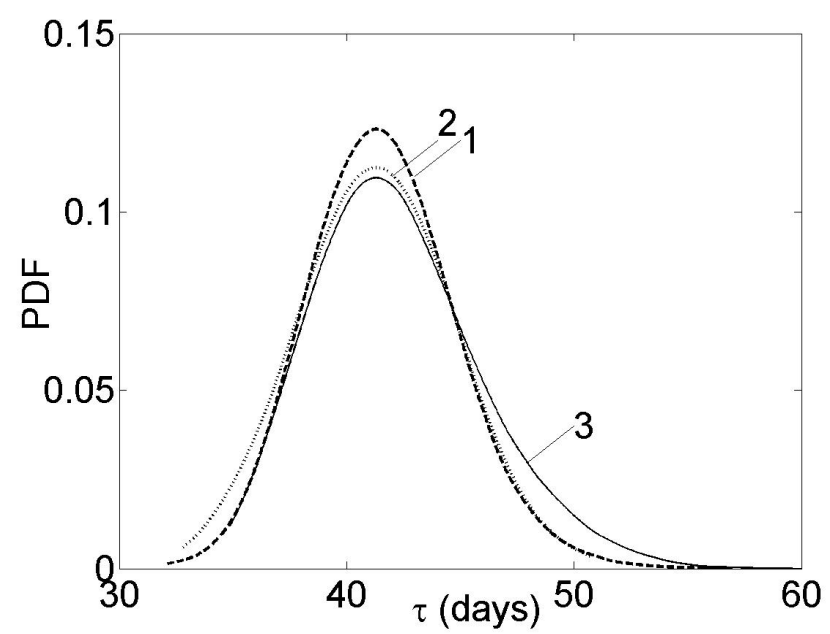

Fig. 6. $\tau$-PDF for the predictability of the first kind with white initial noise (curve-2), and red noise with the correlation radius of $14 \mathrm{~km}$ (curve-1) and $70 \mathrm{~km}$ (curve-3). Here, $I^{2}=0.01 \mathrm{~m}^{2} / \mathrm{s}^{2}$ and $\varepsilon_{0}^{2}=0.5$.

\subsection{Perturbations to open boundary condition}

The normal velocity along the open boundary $\left(u_{b}\right)$ is perturbed by

$u_{b}^{\prime}=B(t) \sin \left(\pi y / L_{y}\right)$,

where $B(t)$ is white noise with zero mean and variance of $\delta^{2}$. The velocity perturbation $\left(u_{b}^{\prime}\right)$ affects inflow and outflow across the open boundary.

\section{Predictability of the first kind}

Keeping wind forcing (2) and open boundary condition (3) accurate, and perturbing initial condition (4) with white or red noise represented by two parameters (variance of initial error $I^{2}$ and correlation radius $R$ ), the predictability of the first kind can be investigated with various combinations of $\left(I^{2}, R, \varepsilon_{0}^{2}\right)$. In all our numerical studies here and hereafter, perturbed model trajectories start from the basin of chaotic attractor, i.e. from the original initial condition (4).

For a given tolerance level $\varepsilon_{0}^{2}(0.5)$, the $\tau$-PDF is close to Gaussian shape (skewness and kurtosis equal to 0.08 and 2.95 , respectively) for the white noise $(R=0$, curve- 1 in Fig. 6). Clearly, any model trajectory starting from the attractor basin does not leave the attractor and the IPT statistics is only determined by the stability of this chaotic attractor.

When the correlation radius $R$ increases, the volume in the phase space covered by the initial error increases (Chu and Ivanov, $2005 \mathrm{a}^{1}$ ), and the two-attractor structure of the reference circulation occurs. For a given error intensity, $I^{2}=0.01 \mathrm{~m}^{2} \mathrm{~s}^{-2}$, the $\tau$-PDF is near Gaussian (curve- 1 in Fig. 6) for $R=0 \mathrm{~km}$, non-Gaussian (curves- 2 in Fig. 6, skewness of 0.58 ) for $R=14 \mathrm{~km}$, and non-Gaussian with a tail 

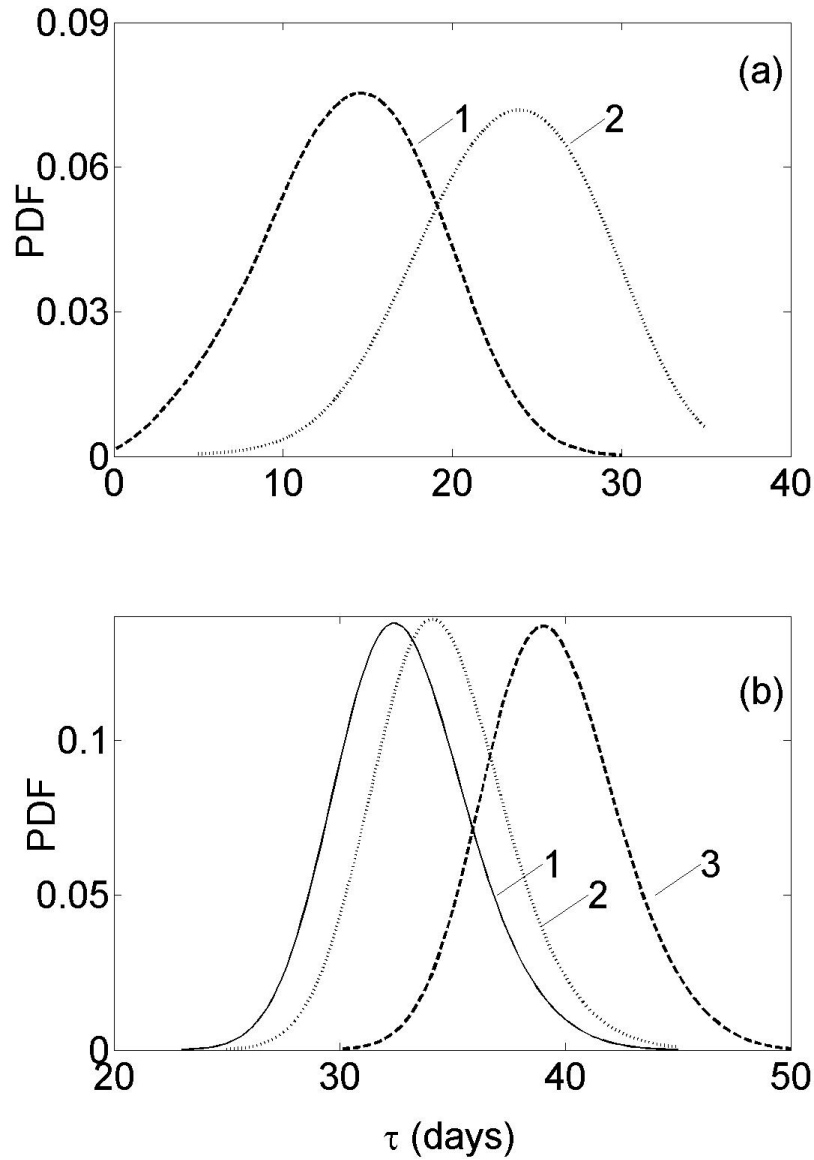

Fig. 7. $\tau$-PDF for the predictability of the second kind (wind forcing) with a given wind error intensity $\left(\sigma^{2}=42 \mathrm{~m}^{2} \mathrm{~s}^{-2}\right)$ and various tolerance levels $\left(\varepsilon_{0}^{2}\right)$ : (a) small tolerance levels: 0.005 (curve-1), 0.01 (curve-2), and (b) intermediate and large tolerance levels: 0.05 (curve-1), 0.1 (curve-2) and 0.5 (curve-3).

stretching to large IPT domain (curves-3 in Fig. 6, skewness of 0.80 ) for $R=70 \mathrm{~km}$.

\section{Predictability of the second kind}

Keep the initial condition (4) accurate, and perturb the wind forcing (2) or open boundary condition (3) with white noises. The noise intensity is $\sigma^{2}$ for the wind forcing and $\delta^{2}$ for the open boundary condition. The second kind of predictability (Lorenz, 1982) can be investigated with various combinations of $\left(\sigma^{2}, \varepsilon_{0}^{2}\right)$ and $\left(\delta^{2}, \varepsilon_{0}^{2}\right)$. Since the predictability of high-resolution regional models depends crucially on the wind forcing (Robinson et al., 1996; Chu et al., 1999a, b, c; Burillo et al., 2002 and others) and the specified normal velocity at the open boundaries, and since errors in the open boundary condition cause a rapid decrease of model predictability skill (Chu, 1999; Jiang and Malanotte-Rizzoli, 1999), investigation of the second kind of predictability is of great importance for regional ocean modeling.

\subsection{Residence time and $\tau$-PDF}

In contrast to the predictability of the first kind, analysis of the second kind of predictability is more difficult because of the multi-attractor system. "Noise induced escape" occurs when a model trajectory switches over attractors (Anishenko, 1997). Therefore, in addition to IPT, the residence time $\left(\tau_{\text {res }}\right)$ should be used with the definition of a maximum time period for a perturbed trajectory keeping in the same attractor.

In general, the residence time depends on the basin volume of the attractor, initial position of model trajectory at the attractor, dynamical attractor stability and noise intensity $\left(\sigma^{2}\right.$ or $\delta^{2}$ ) (Kaneko, 1998). Since there is no common method to estimate $\tau_{\text {res }}$ on the attractor for a high-dimensional dissipative dynamical system (Kaneko, 1998), a phenomenological analysis of relationship between $\tau$ and $\tau_{\text {res }}$ is suggested.

For a small $\varepsilon_{0}^{2}$, it is expected that

$\tau<\tau_{\text {res }}$,

which shows that the model losses the predictability skill before the model trajectory leaves the occupied attractor. The $\tau$-PDF is determined by the stability of a single attractor and probably should be near Gaussian type.

For a sufficiently large $\varepsilon_{0}^{2}$, it is expected that

$\tau>\tau_{\text {res }}$,

which shows that the model keeps the predictability skill after the model trajectory leaves the occupying attractor. This leads to the change of the $\tau$-PDF structure. Therefore, switch among attractors can be identified through analyzing the change of $\tau$-PDF structure with increasing $\varepsilon_{0}^{2}$.

The proposed methodology, of course, can not determine the residence time $\tau_{\text {res }}$ and the number of trajectories switching among attractors. However, it determines the change of the $\tau$-PDF structure that links to the duration of the perturbed trajectory along different attractors. Besides, it uniquely identifies the trajectory switching from the chaotic attractor to the limit cycles when the stochastic perturbation is added to external forcing.

\subsection{Perturbations to wind forcing}

The two parameters $\sigma^{2}$ and $\varepsilon_{0}^{2}$ determine the $\tau$-PDF structure. For small tolerance level $\left(\varepsilon_{0}^{2}<0.01\right)$ and strong wind perturbations $\left(\sigma^{2}>40 \mathrm{~m}^{2} \mathrm{~s}^{-2}\right)$, the $\tau$-PDF is nearly symmetric with large variance of IPT. The kurtosis and skewness are approximately 3.1 and 0.1 (Fig. 7a).

With increase of $\varepsilon_{0}^{2}(>0.025)$, the $\tau$-PDF becomes asymmetric with smaller IPT variance and a long tail stretching to large IPT domain (Fig. 7b). The skewness is 0.60 for curve- 1 and 0.75 for curve- 3 . This shows that the tolerance level $\varepsilon_{0}^{2}$ is a controlling parameter for model predictability skill. The $\tau$ PDF is symmetric for small $\varepsilon_{0}^{2}$ and asymmetric for large $\varepsilon_{0}^{2}$. For a relatively large tolerance level $\left(\varepsilon_{0}^{2} \geq 0.05\right)$, the asymmetry of the $\tau$-PDF structure increases (longer tail toward large IPT domain) with the increase of noise intensity $\sigma^{2}$ (Fig. 8). 


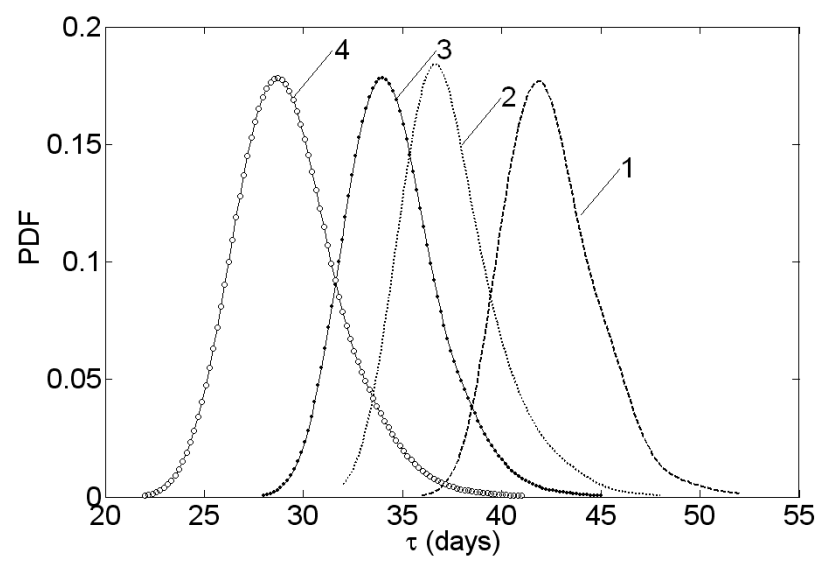

Fig. 8. $\tau$-PDF for the predictability of the second kind (wind forcing) with a given tolerance level $\varepsilon_{0}^{2}=0.1$ with various wind error intensity: $\sigma^{2}=14 \mathrm{~m}^{2} \mathrm{~s}^{-2}$ (curve-1), $28 \mathrm{~m}^{2} \mathrm{~s}^{-2}$ (curve-2), $42 \mathrm{~m}^{2} \mathrm{~s}^{-2}$ (curve-3), and $56 \mathrm{~m}^{2} \mathrm{~s}^{-2}$ (curve-4).

The extra-successful predictions (Chu et al., 2002c) corresponding to the PDF tail (toward the large IPT domain) are not outliers since they share the same statistics with other predictions with short and intermediate IPTs.

Another important result is that the multi-attractor structure of the modeled circulation does not necessary lead to the multi-modal $\tau$-PDF since the perturbed trajectory can switch among different attractors. This is different from a recent result reported by Miller and Ehret (2002) that if the initial error co-variances are large enough such that a given perturbed trajectory has high possibility to start from different basin attractions, the initial Gaussian $\tau$-PDF will evolve into a bimodal $\tau$-PDF.

Existence of a tail stretching into large IPT domain is the most common feature of $\tau$-PDF for short and intermediate forecasts (Fig. 8). Growth of the noise intensity $\sigma^{2}$ increases the asymmetry of the $\tau$-PDF. For example, the skewness is 0.38 for $\sigma^{2}=14 \mathrm{~m}^{2} \mathrm{~s}^{-2}$ (curve-1) and 0.96 for $\sigma^{2}=56 \mathrm{~m}^{2} \mathrm{~s}^{-2}$ (curve-4). Chu and Ivanov (2005a) ${ }^{1}$ pointed out that the Weibull distribution is the best fit for the $\tau$-PDFs illustrated in Fig. 8. Therefore, the extremely successful predictions (Chu et al., 2002b) corresponding to the $\tau$-PDF tails are not outliers since they share the same statistics with the usual predictions.

The $\tau$-PDF is nearly symmetric for small $\varepsilon_{0}^{2}$ and asymmetric for large $\varepsilon_{0}^{2}$. Loss of the $\tau$-PDF symmetry occurs as $\varepsilon_{0}^{2}>0.025$. This indicates that variation of the tolerance level $\varepsilon_{0}^{2}$ may lead to a drastic change of statistics for measuring the model predictability skill. A set of structural change of the $\tau$-PDF with the increase of $\varepsilon_{0}^{2}$ may be considered as the one-step bifurcation sequence:

$\tau$-PDF symmetry $\rightarrow \tau$-PDF asymetry.

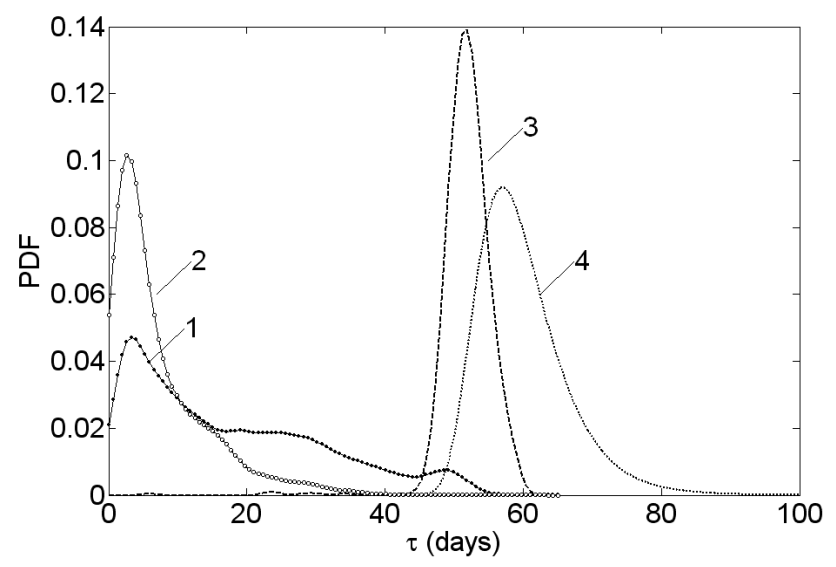

Fig. 9. $\tau$-PDF for the second kind of predictability (open boundary) with a given open boundary error intensity $\left(\delta^{2}=0.2 \mathrm{~m}^{2} \mathrm{~s}^{-2}\right)$ and various tolerance levels $\left(\varepsilon_{0}^{2}\right): 0.005$ (curve-1), 0.01 (curve-2), 0.1 (curve-3) and 0.5 (curve-4).

\subsection{Perturbations to open boundary condition}

For small tolerance level $\left(\varepsilon_{0}^{2} \leq 0.01\right)$, the $\tau$-PDF is already asymmetric with a maximum value at small IPT domain (about 5 days, i.e., the most probable IPT) and a tail stretching to large IPT domain up to 40-50 days (curves- 1 and curve-2 in Fig. 9). The skewness is 3.8 for $\varepsilon_{0}^{2}=0.005$ (curve1) and 3.9 for $\varepsilon_{0}^{2}=0.01$ (curve-2). The estimated mean residence time is about 10 days, the perturbed model trajectories departure quickly from the chaotic attractor to the small amplitude limit cycle. With increase of $\varepsilon_{0}^{2}(>0.025)$, the IPT variance and the degree of $\tau$-PDF asymmetry reduces (curves-3 and curve-4 in Fig. 9). The skewness decreases to $0.7-0.8$. This is caused by the perturbed trajectories having time to return to the chaotic attractor from the limit cycle.

For some values of $\left(\varepsilon_{0}^{2}, \delta^{2}\right)$, there exist "outliers" - predictions with statistical properties differing considerably from those of the prediction ensemble. For example, when $\varepsilon_{0}^{2}=0.1$, $\delta^{2}=0.3 \mathrm{~m}^{2} \mathrm{~s}^{-2}$, the $\tau$-PDF shows a complex structure with two distinct features for $\tau<40$ day and $\tau>40$ day (Fig. 10a). The $\tau$-PDF in Fig. 10a can be represented as the combination of two $\tau$-PDFs: (a) uniform distribution (shown in Fig. 10b) for $12 \mathrm{~h} \leq \tau \leq 39$ days and (b) near Gaussian distribution with the mean and variance equal to 50 day and $7.5 \mathrm{day}^{2}$, respectively. Therefore, the predictions for $\tau<40$ day may be identified as outliers.

A set of structural change of the $\tau$-PDF with the increase of $\varepsilon_{0}^{2}$ may be considered as the two-step bifurcation sequence:

asymetric $\tau$-PDF with large variance $\rightarrow$

$\rightarrow$ symmetric $\tau$-PDF + outliers $\rightarrow$

$\rightarrow$ asymetric $\tau$-PDF with small variance.

Existence of bifurcation sequences (17) and (18) should be checked in atmospheric and oceanic models. 

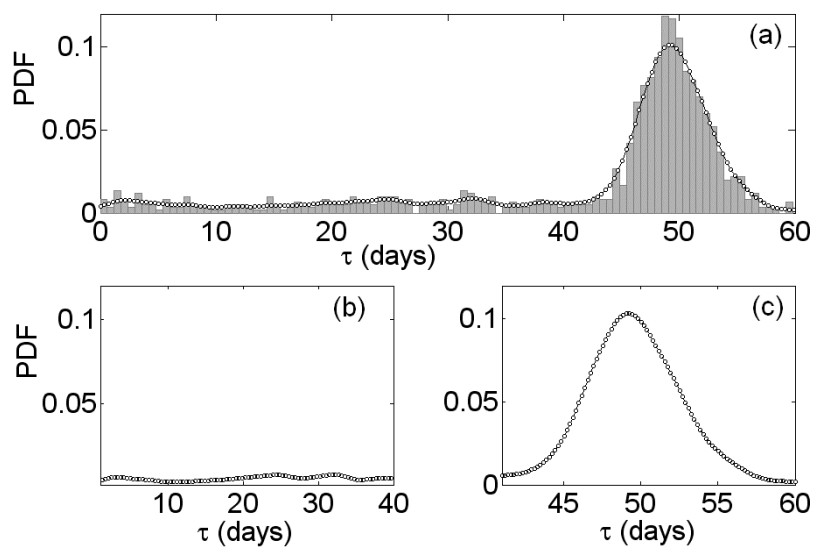

Fig. 10. "Outliers" - forecasts with $\delta^{2}=0.3 \mathrm{~m}^{2} \mathrm{~s}^{-2}, \varepsilon_{0}^{2}=0.1$ : (a) $\tau$ histogram computed for the perturbed open boundary, (b) best fit to the homogeneous distribution for $\tau<40$ day, and (c) best fit to Gaussian distribution for $\tau>40$ day.

\section{Conclusions}

(1) Several predictability regimes in two-attractor circulations (the chaotic attractor and small-amplitude limit cycle) reproduced by a numerical model have been identified in a semi-closed basin with flat bottom. The $\tau$-PDF is nonGaussian for intermediate and large amplitude prediction errors, and near Gaussian for small amplitude prediction errors.

(2) For the predictability of the first kind, the error evolution depends strongly on the correlation radius of the initial error and its variance. If the initial co-variances and correlation radius are large enough so that there is large possibility that a given perturbed model trajectory does not start from the same attractor basin as the mean ensemble one, the $\tau$ PDF is asymmetric (non-Gaussian) with a tail stretching into large IPT domain.

(3) The stochastic perturbations added to the wind forcing and open boundary condition lead to switch of the perturbed model trajectories over attractors (i.e., the noise-induced escape). It is found that the perturbed model trajectories switch from the chaotic attractor to the limit cycles and switch back. Relationship between the residence time at the attractor and IPT determines the shape of $\tau$-PDF. For $\tau<\tau_{\text {res }}$ perturbed model trajectories do not have time to switch from the chaotic attractor to the limit cycle. This causes the $\tau$-PDF near Gaussian. For $\tau \sim \tau_{\text {res }}$ the perturbed model trajectories can be either in the initial chaotic attractor or in the limit cycle. This causes the bimodal (not often) or asymmetric nonGaussian (more often) $\tau$-PDF. For $\tau>\tau_{\text {res }}$, the perturbed trajectories have sufficient time to switch to the limit cycle, and cause asymmetric $\tau$-PDF.

(4) The perturbed model trajectories attracted to the limit cycle share the same statistical properties as the trajectories still along the initial chaotic attractor. In general, the numerical computations do not indicate the outlier feature of predictions except for some forecasts realized for specific com- binations of the tolerance level and variance of perturbations added to the open boundary condition.

(5) Two different bifurcation sequences in changes of $\tau$ PDF are found with increase of $\varepsilon_{0}^{2}$. For uncertain wind forcing, one-step bifurcation is found with symmetric $\tau$-PDF changing to asymmetric $\tau$-PDF. For uncertain open boundary conditions, a more complicated two-step bifurcation is found in the model predictability skill.

\section{Appendix A}

Spectral decomposition

A two-dimensional compressible circulation $(u, v)$ can be represented by

$u=-\frac{\partial \Psi}{\partial y}+\frac{\partial \Phi}{\partial x}, v=\frac{\partial \Psi}{\partial x}+\frac{\partial \Phi}{\partial y}$,

where $\Psi$ and $\Phi$ are the geostrophic streamfunction and velocity potential, respectively. Let $\left(\Gamma, \Gamma^{\prime}\right)$ be the rigid and open boundaries. The geostrophic streamfunction $\Psi$ is decomposed into (Eremeev et al., 1992; Chu et al., 2003)

$\Psi(x, y, t)=\Psi_{H}(x, y, t)+\sum_{k=1}^{\infty} Z_{k}(t) \Psi_{k}(x, y)$,

where $\left\{\Psi_{k}\right\}$ are the eigen-functions of the spectral problem,

$\Delta \Psi_{k}(x, y)=-\lambda_{k} \Psi_{k}(x, y),\left.\quad \Psi_{k}\right|_{\Gamma \cup \Gamma^{\prime}}=0$,

with $\lambda_{k}$ the eigen-values of the horizontal Laplacian $(\Delta)$. The component $\Psi_{H}$ is the harmonic function satisfying the boundary conditions

$\left.\Psi_{H}\right|_{\Gamma}=0,\left.\quad \Psi_{H}\right|_{\Gamma^{\prime}}=\Psi_{b}$,

where $\Psi_{b}$ is computed from knowledge of the normal velocity at open boundary.

The velocity potential $\Phi$ is decomposed into

$\Phi=\sum_{m=1}^{\infty} B_{m}(t) \Phi_{m}(x, y)$,

where $\left\{\Phi_{m}\right\}$ are the eigen-functions of the spectral problem

$\Delta \Phi_{m}(x, y)=-\mu_{m} \Phi_{m}(x, y)$,

$\left.\Phi_{m}\right|_{\Gamma^{\prime}}=0$,

$\left.\mathbf{n} \cdot \nabla \Phi_{m}\right|_{\Gamma}=0$,

where $\mathbf{n}$ is the unit vector normal to $\Gamma^{\prime} ;\left\{\mu_{m}\right\}$ are the eigenvalues.

The basis functions $\left\{\Psi_{k}, \Phi_{m}\right\}$ generate the phase space which can be used for the spectral analysis of ocean circulation in a basin with real coastlines. Since the surface elevation is small comparing to the water depth $H$,

$\zeta / H \ll 1$

the quasi-geostrophic approximation is reasonable for the flow reproduced in the present study (Pedlosky, 1987). Thus, in the main text, only the geostrophic streamfunction $\Psi$ is considered. 
Acknowledgements. This study was supported by the Office of Naval Research, Naval Oceanographic Office, and the Naval Postgraduate School. We appreciate the helpful comments from two anonymous reviewers that substantially improved the manuscript.

Edited by: N. W. Watkins

Reviewed by: two referees

\section{References}

Anishenko, V. S.: Attractor dynamical system, Appl. Non. Dyn., 5 , 1, 109-127, 1997.

Auclair, F., Marsaleix, P., and De Mey, P.: Space-time structure and dynamics of the forecast error in a coastal circulation model of the Gulf of Lions, Dyn. Atmos. Ocean, 36, 309-346, 2003.

Berloff, P. S. and McWilliams, J. C.: Large-scale, low-frequency in wind-driven ocean gyres, J. Phys. Oceanogr., 29, 1925-1949, 1999.

Blumberg, A. F. and Mellor, G. L.: A description of a threedimensional coastal ocean circulation model, in: Three Dimensional Coastal Ocean Models, Amer. Geophys. Union, 1-16, 1987.

Buizza, R., Miller, M. J., and Palmer, T. N.: Stochastic simulation of model uncertainties in the ECMWF ensemble prediction system, Q. J. Meteorol. Soc., 125, 2887-2908, 1999.

Burillo, I. A., Caniaux, G., Gavart, M., De Mey, P., and Baraille, R.: Assessing ocean-model sensitivity to wind forcing uncertainties, Geophys. Res. Lett., 29, 18, 5-1-5-4, 2002.

Charney, J. and de Vore, J.: Multiple flow equilibria in the atmosphere and blocking, J. Atm. Sci., 36, 1205-1216, 1979.

Chu, P. C.: Two kinds of predictability in the Lorenz system, J. Atmos. Sci., 56, 1427-1432, 1999.

Chu, P. C. and Ivanov, L. M.: Linear and nonlinear perspectives of forecast error estimate using the first passage time, in: Proc. "The 82nd American Meteorological Society Annual Meeting, The Symposium on Observations, Data Assimilation and Probabilistic Prediction", Orlando, 127-132, 2002.

Chu, P. C., Fan, C. W., and Ehret, L. L.: Determination of open boundary conditions with an optimization method, J. Atmos. Oceanic Technol., 14, 723-734, 1997.

Chu, P. C., Lu, S. H., and Liu, W. T.: Uncertainty of the South China Sea prediction using NSCAT and NCEP winds during tropical storm Ernie 1996, J. Geophys. Res., 104, $11273-11$ 289, 1999a.

Chu, P. C., Lu, S. H., and Chen, Y. C.: A coastal atmosphere-ocean coupled system (CAOCS) evaluated by an airborne expandable bathythermograph survey in the South China Sea, May 1995, J. Oceanogr., 55, 543-558, 1999b.

Chu, P. C., Edmons, N. L., and Fan, C. W.: Dynamical mechanisms for the South China Sea seasonal circulation and thermohaline variability, J. Phys. Oceanogr., 29, 2971-2989, 1999c.

Chu, P. C., Chen, Y. C., and Liu, W. T.: Uncertainty of the South China Sea prediction using NSCAT and NCEP winds during Tropical Storm Ernie1996, J. Geophys. Res., 104, 1273-1289, 1999d.

Chu, P. C., Lu, S., and Chen, Y.: Evaluation of the Princeton Ocean Model using South China Sea Monsoon Experiment (SCSMEX) data, J. Atm. Ocean. Tech., 18, 1521-1539, 2001.

Chu, P. C., Ivanov, L. M., Margolina, T. M., and Melnichenko, O. V.: On probabilistic stability of an atmospheric model to various amplitude perturbations, J. Atm. Sci., 59, 2860-2873, 2002a.
Chu, P. C., Ivanov, L. M., Kantha, L., Melnichenko, O. V., and Poberezhny, Y.: Power law decay in model predictability skill, Geophys. Res. Lett., 29 (15), 10.1029/2002GLO14891, 2002 b.

Chu, P. C., Ivanov, L. M., Margolina, T. M., Korzhova, T. P., and Melnichenko, O. V.: Analysis of sparse and noisy ocean current data using flow decomposition, Part-1, Theory, J. Atm. Ocean. Tech., 20, 478-491, 2003.

Denholm-Price, J. C.: Can an ensemble give anything more than Gaussian probabilities?, Non. Proc. Geophys., 10, 469-475, 2003,

SRef-ID: 1607-7946/npg/2003-10-469.

Eremeev, V. N., Ivanov, L. M, Kochergin, S. V., and Melnichenko, $\mathrm{O}$. V: Seasonal variability and types of currents in the upper layer of the Black Sea, Sov. J. Phys. Oceanogr., 3, 193-208. 1992.

Good, P. I.: Re-Sampling Methods, A Practical Guide to Data Analysis, Birkhauser, Boston-Basel-Berlin, 425, 1996.

Guchenheimer, J. and Holmes, Ph.: Nonlinear Oscillations, Dynamical Systems, and Bifurcations of Vector Fields, Springer, Berlin, 415, 1983.

Ivanov, L. M., Kirwan, A. D., and Melnichenko, O. V.: Prediction of the stochastic behavior of nonlinear systems by deterministic models as a classical time-passage probabilistic problem, Non. Proc. Geophys., 1, 224-233, 1994,

SRef-ID: 1607-7946/npg/1994-1-224.

Jiang, S. and Malanotte-Rizzoli, P.: On the predictability of regional oceanic jet stream: the impact of model errors at the inflow boundary, J. Mar. Res., 57, 641-669, 1999.

Kaneko, K.: On the strength of attractors in a high-dimensional system, Milnor attractor: Milnor attractor network, robust global attraction, and noise-induced selection, Physica D, 124, 322-344, 1998.

Latin hypercube sampling tool: http://www.mathepi.com/epitools/ lhs/nrpage.html, 2001.

Lorenz, E. N.: Atmospheric predictability experiments with a large numerical model, Tellus, 34, 505-513, 1982.

Lozano, C. J., Robinson, A. R., Arango, H. G., Gangopadhyay, A., Sloan, N. Q., Haley, P. J., and Leslie, W. G.: An interdisciplinary ocean prediction system: assimilation strategies and structured data models, in: Modern Approaches to Data Assimilation in Ocean Modeling, edited by: Malanotte-Rizzoli, P., Elsevier, 413-456, 1996.

Masuda, S., Akitomo, K., and Awaji, T.: Effects stratification and bottom topography on the Kuroshio path variation south of Japan, Part 1: Dependence of the path selection on velocity, J. Phys. Oceanogr., 29, 2419-2431, 1999.

Meacham, S. P. and Berloff, P. S.: Barotropic, wind-driven circulation in a small basin, J. Mar. Res., 55, 523-563, 1997.

Miller, R. N. and Ehret, L. L.: Ensemble generation for models of multi-modal systems, Mon. Wea. Rev., 130, 2313-2333, 2002.

Molteni, F.: On the dynamics of planetary flow regimes, Part II: Results from a hierarchy of orographically forced models, J. Atmos. Sci., 53, 1972-1992, 1996.

Pedlosky, J.: Geophysical Fluid Dynamics, 2nd Edition, SpringerVerlag, New York, 710, 1987.

Robinson, A. R., Arango, H. G., Warn-Varnas, A., Leslie, W. G., Miller, A. J., Haley, P. J., and Lozano, C. J.: Real-time regional forecasting, in: Modern Approaches to Data Assimilation in Ocean Modeling, edited by: Malanotte-Rizzoli, P., Elsevier, 337-409, 1996.

Sabel'feld, K.: Monte-Carlo Methods in Boundary Value Problems, Springer-Verlag, 274, 1991. 
Schmeits, M. J. and Dijkstra, H. A.: Physics of the 9-month variability in the Gulf Stream region: combining data and dynamical systems analyses, J. Phys. Oceanogr., 30, 1967-1987, 2000.

Sirovich, L.: Chaotic dynamics of coherent structures, Physica D, 37, 126-145, 1989.

Sura, P., Fraedrich, K., and Lunkeit, F.: Regime transitions in a stochastically forced double-gyre model, J. Phys. Oceanogr., 31, 411-426, 2001.
Toth, Z. and Kalnay, E.: Ensemble forecasting at NCEP: the breeding method, Mon. Wea. Rev., 125, 3297-3318, 1997.

Wright, P. B.: An atlas based on the COADS dataset: Fields of mean wind, cloudiness and humidity at the surface of global ocean, Report 14, Max-Plank-Institute for Meteorology, 68, 1988. 